\title{
BMJ Open Global epidemiology of acute generalised peritonitis: a protocol for a systematic review and meta-analysis
}

\author{
Joel Noutakdie Tochie (D) , ${ }^{1}$ Ndip Valirie Agbor (D) , ${ }^{2}$ Tianyi Tianyi Frank Leonel, ${ }^{3}$ \\ Aime Mbonda, ${ }^{4}$ Desmond Aji Abang, ${ }^{5}$ Celestin Danwang (iD ${ }^{6,7}$
}

To cite: Tochie JN, Agbor NV, Frank Leonel TT, et al. Global epidemiology of acute generalised peritonitis: a protocol for a systematic review and meta-analysis. BMJ Open 2020;10:e034326. doi:10.1136/ bmjopen-2019-034326

- Prepublication history and additional material for this paper are available online. To view these files, please visit the journal online (http://dx.doi. org/10.1136/bmjopen-2019034326).

Received 15 September 2019 Revised 16 December 2019 Accepted 18 December 2019

Check for updates

(C) Author(s) (or their employer(s)) 2020. Re-use permitted under CC BY-NC. No commercial re-use. See rights and permissions. Published by BMJ.

For numbered affiliations see end of article.

Correspondence to Dr Joel Noutakdie Tochie; joeltochie@gmail.com

\section{ABSTRACT}

Introduction Globally, acute generalised peritonitis (AGP) is a common medical and surgical emergency which is a major contributor to non-trauma deaths despite improvements in diagnosis and surgical and intensive care management. In order to determine the global burden of AGP, geared at tailoring key interventions to curb its morbidity and mortality, we proposed this first ever systematic review and meta-analysis to estimate the contemporary prevalence, and to determine the most frequent AGP and the case fatality rate of AGP, at the global scene.

Methods and analysis We intend to search African Journals Online, Americana em Ciências da Saúde, Citation index, EMBASE, Global Index Medicus, Literatura Latino Africa Index Medicus, Medline and Scientific Electronic Library Online databases from 1 January 2009 to 31 July 2019 to identify studies that reported the prevalence, types of AGP, and case fatality rate of AGP in the global population without any language restrictions. Study selection, data extraction and risk of bias assessment will be conducted independently at each level by a pair of independent investigators. Random-effects metaanalysis will be used to pool studies judged to be clinically homogeneous. The presence of heterogeneity will be evaluated using the $\chi^{2}$ test on Cochrane's $Q$ statistic and quantified with the $\mathrm{I}^{2}$ statistics. Publication bias will be evaluated statistically and visually using the Egger's test and funnel plots, respectively. Findings will be reported and compared by countries, WHO regions and globally. Ethics and dissemination Since this study will be based on published data, it does will not require an ethical approval. The findings will be published in a scientific peer-reviewed journal. They will also be presented at scientific conferences and to relevant public health actors. PROSPERO registration number CRD42019143331.

\section{BACKGROUND}

Acute generalised peritonitis (AGP) is a potentially life-threatening intra-abdominal pathology worldwide which partly manifests as an acute abdomen. ${ }^{12}$ AGP is the most frequent cause of acute abdomens ${ }^{34}$ requiring broadspectrum antibiotics and source control with laparotomy or laparoscopic surgery for definitive cure, ${ }^{56}$ except in case of spontaneous peritonitis which is a medical emergency

\section{Strengths and limitations of this study}

To the best of our knowledge, this systematic review and meta-analysis will be the first reporting the global epidemiology of acute generalised peritonitis (AGP).

- This current review will include recent studies, hence, it will inform and guide policy makers in decision making on a global health perspective related to AGP.

- The absence of language restriction in the methods will allow enrolment of the maximum number of studies published on the topic.

- A limited number of studies on the topic from some continents, regions or countries could lead to an underestimation of the true global burden of AGP.

treated with antibiotics only. ${ }^{7}$ Furthermore, AGP is a major contributor to non-trauma deaths in all emergency settings, and the second leading cause of sepsis in critically ill patients. ${ }^{8}{ }^{9}$ Its pathophysiology involves an inflammatory process of the peritoneum caused by an infectious or chemical agent which irritates the peritoneal cavity by either haematogenous spread of infection from another body part to the peritoneal cavity, or by perforation of an intra-abdominal hollow viscus with resultant spillage of bacteria into the peritoneal cavity. ${ }^{3} 4710$ A robust local inflammatory response then stems in to control the inflammation which is mostly infectious in nature. If not treated in time, it spreads to the systemic circulation leading to bacteraemia, septicaemia, septic shock, multiorgan dysfunction and ultimately death. ${ }^{5}$

AGP affects about 9.3 patients per 1000 hospital admissions ${ }^{11}$ and requires adequate preoperative resuscitation to avert high perioperative morbidity and mortality. ${ }^{12} 13$ Although AGP is one of the most frequent surgical emergencies at the global level, ${ }^{814}$ it has been reported to disproportionately affect low-income and middle-income countries 
especially the sub-Saharan African (SSA) region both in terms of prevalence ${ }^{915}$ and mortality rates. ${ }^{16-18}$ The most common types of AGP in a recent series of 305 cases in SSA were perforated gastroduodenal ulcers, perforated appendicitis and typhoid ileal perforation. ${ }^{9}$ The postoperative mortality rates of AGP vary between $8.4 \%$ and $34 \%$ from isolated studies. ${ }^{145919}$ Various aetiologies of AGP that contribute to the death toll in SSA have been described as follows; typhoid perforation of the terminal ileum $(34.7 \%)$, postoperative peritonitis $(19.5 \%)$ and peptic ulcer perforation $(15.2 \%)$, perforated appendicitis $(8.7 \%)$, and perforation of the sigmoid colon $(8.7$ \%). ${ }^{9}$ AGP also poses the problems of hypovolaemic shock, and postoperative complications like surgical site infections, chest infection, intestinal obstruction, postoperative peritonitis, thromboembolic diseases, incisional hernia, wound dehiscence, enterocutanous fistulas, reoperation and prolonged hospital stay. ${ }^{9} 1920$ AGP increases the risk of perioperative morbidity and mortality, and it incurs a high economic burden on affected patients, families and the health system as a whole. ${ }^{5}$ It is worth mentioning that AGP can also be iatrogenic after some medical procedures such as continuous ambulatory peritoneal dialysis. ${ }^{21}$ Despite the tremendous progress in laboratory tests, imagining studies, perioperative resuscitation, surgical techniques and intensive care treatment, the management of AGP is still highly complex and represents a major challenge for all surgical and anaesthetist teams. ${ }^{5} 622$

The above synthesis elucidates that AGP still remains a potentially fatal affliction. With a wide variety of aetiologies, advances in therapies, divergent prevalence data and mortality rates of AGP, it is important to set priorities, funding for surgical interventions and healthcare planning. Hence, it is necessary to have accurate data on the global epidemiology of AGP in order to curb its burden. Hence, to fill these knowledge gaps on the global epidemiology of AGP, we propose this systematic review and meta-analysis protocol to critically synthesise contemporary evidence on the occurrence of AGP in the world. This study will provide the best understanding of the scope of this public health concern and is intended to both inform and draw the attention of surgeons, researchers, public health authorities and governments to this frequent cause of acute abdomen.

\section{Review questions}

1. What is the global prevalence of AGP and its different types?

2. What is the deadliest AGP in the world and the global case fatality rate of AGP?

\section{Objectives}

This systematic review and meta-analysis aims to:

1. Determine the global prevalence of AGP and its different types.

2. Determine global and case fatality rate per type of AGP.
METHODS AND ANALYSIS

\section{Design and registration}

This systematic review and meta-analysis will be carried out using Preferred Reporting Items for Systematic Reviews and Meta-Analysis (PRISMA), ${ }^{23}$ reported according to PRISMA Protocols ${ }^{24}$ as illustrated in the online supplementary file $1 .^{25}$

\section{Criteria for considering studies for this review}

1. Types of studies: We will consider cross-sectional, case-control and cohort studies. We will exclude case reports, letters, comments, editorials, experimental studies and case series with less than 30 participants.

2. Types of participants: We will consider studies conducted on AGP in all age groups (newborns, toddlers, children, adolescents, adults and the elderly) all over the world. Studies conducted in patients selected based on the presence of a specific disease or a condition like HIV infection, chronic liver disease or chronic kidney disease will be excluded.

3. Type of interventions: medical (antibiotic therapy) or surgical (laparotomy or laparoscopic) treatment.

4. Types of outcomes: We will consider studies reporting the following outcomes with enough data to compute these estimates: prevalence, types (primary, secondary and tertiary) and the case-fatality rate of AGP. AGP will categorised into primary, secondary or tertiary AGP. ${ }^{20}$ The primary outcome will be the prevalence of AGP defined as the cases of AGP divided by the total population at a given time period per included study. The secondary outcomes will be the type of AGP and its case fatality rate. Primary AGP also called spontaneous bacterial peritonitis will be defined as community acquired or nosocomially acquired peritonitis caused by haematogenous spread of bactieria or fungi to the peritoneal cavity. Secondary AGP will be defined as peritonitis resulting from an outburst of intra-abdominal viscera such as the stomach, gall bladder, small or large bowels, and appendix with resultant spillage of bacteria from the gastrointestinal tract into the peritoneal cavity. ${ }^{3}$ Tertiary peritonitis will be defined as peritoneal irritation caused by a penetrating abdominal injury with gastric, intestinal or biliary lesions leading to spillage of bacteria from the gastrointestinal tract into the peritoneal cavity, ${ }^{20}$ or as an intra-abdominal infection which recurs or persists 48 hours following successful and adequate surgical management of secondary peritonitis. ${ }^{10}$ The case fatality rate of AGP will be the number of death due to peritonitis divided by the number of patients admitted for peritonitis. Studies with no detailed definition of AGP or a different classification system of AGP to that mentioned above would be described qualitatively.

\section{Search strategy for identifying relevant studies}

The following databases will be considered: African Journals Online, Americana em Ciências da Saúde, Citation index, EMBASE, Global Index Medicus, Literatura Latino 
Africa Index Medicus, Medline and Scientific Electronic Library Online from 1 January 2009 to 31 July 2019. We will search records regardless of language of publication. The search strategy for some databases is illustrated in the online supplementary file 2 . The reference list of the eligible articles and relevant reviews will be manually searched to identify additional studies. Key search terms will include: 'epidemiology', 'peritonitis', 'type' and 'case fatality rate'. Grey literature will also be searched through theses, conference proceedings, book chapters, and governmental and non-governmental organisations reports. We are working hand in glove with a Librarian who designed the search strategy and identified a total number of 7952 articles through this search procedure. The Librarian wishes his name to remain anonymous.

\section{Selection of studies for inclusion in the review}

Two investigators (NVA and TTFL) will independently select records based on the titles and abstracts. Any disagreement will be solved by discussion and consensus. Studies in languages other than English or French will be translated by a certified translator and considered for eligibility. These investigators will independently evaluate the full text of the selected records. The agreement between the two investigators will be estimated by Cohen's $\kappa$ coefficient. ${ }^{26}$ Rayyan software will be used for data management in the review selection process.

\section{Methodological quality assessment}

The methodological quality of the finally included studies will be assessed using the Joanna Briggs Institute tool for prevalence studies (online supplementary file 3 ).$^{27}$ The defined questions will be scored with 1 for 'Yes' and 0 for 'No' or 'Unclear'. The total score of each article will be calculated by the sum of its points. Based on this tool, studies will be rated as low risk, moderate risk and high risk with scores $0-3,4-6$ and 7-9, respectively. Discrepancy of risk of bias assessment among the six investigators (JNT, NVA, TTFL, AM, DAA and CD) will be solved by discussion and consensus or by arbitration by CD.

\section{Data extraction and management}

Data extraction will be done independently by five investigators (JNT, NVA, TTFL, AM and CD). Disagreements among each pair of investigators will be solved by discussion or will involve, if necessary, a third review author for arbitration (DAA). Using a pretested form, we will extract: the last name of the first author, year of publication, region, country's name, study area (rural or urban), study setting, country, number of study sites (one site, multicentre), period of inclusion of participants, timing of data collection (retrospective or prospective), mean or median age, male proportion, study design, sample size, delay between diagnosis and treatment ( $<$ or $>24$ hours), American Society of Anesthesiologists grade (I, II, III, IV and V), any type of shock (septic, hypovolaemic or mixed) on admission, the type of AGP (tuberculosis peritonitis, fungal peritonitis, appendicular peritonitis, typhoid ileal peritonitis, peptic ulcer peritonitis, peritonitis due to bowel injury, etc), specific characteristics of the study population (patients with HIV, diabetes mellitus, inflammatory bowel diseases, cancer, organ transplants or any other specific condition), management options (antibiotic therapy, laparotomy, laparoscopy), Mannheim Peritonitis Index prevalence and case fatality rate of AGP. For multicentre studies conducted in different countries, the prevalence, types and case fatality rate of AGP will be reported for the individual countries. The countries will be grouped in regions according to the WHO regional classification: Africa, Americas, Eastern Mediterranean, Europe, South-East Asia and Western Pacific. Where relevant outcome estimates will not be available, we will contact the corresponding author of the study to request the missing information via emails. If no response is obtained from the corresponding author after two sent emails in a time frame of 1 month, the study will be excluded.

\section{Data synthesis and analysis}

Data analyses will be done using the 'meta' package of $\mathrm{R}$ (V.3.2.2). Unadjusted prevalence will be recalculated based on the information of crude numerators and denominators provided by individual studies. We will then perform random-effects meta-analyses. ${ }^{28}$ To keep the effect of studies with extremely small or extremely large prevalence estimates, the variance of the study-specific prevalence will be stabilised with Freeman-Tukey double arcsine transformation before pooling the data. ${ }^{29}$ Heterogeneity will be evaluated by the $\chi^{2}$ test on Cochrane's $Q$ statistics ${ }^{30}$ and quantified by $\mathrm{I}^{2}$ values. ${ }^{31}$ In case heterogeneity is encountered, subgroup analyses will be performed according to the income level of the country, the type of peritonitis, its aetiology and the WHO region. Publication and selection bias will be assessed visually by inspection of the funnel plot. The presence of publication bias will be detected by the formal Egger's test ${ }^{32}$ A value of $p<0.10$ on Egger's test will be considered indicative of statistically significant publication bias.

\section{Protocol development and potential amendments}

No funder, sponsor or institution was involved in the conception and design of this protocol. We do not plan to make any changes to this protocol. However, if substantial changes occur during the review, they will be reported in the published results.

\section{Patient and public involvement}

Patients and public were not involved in the conception and design of this protocol.

\section{Author affiliations}

${ }^{1}$ Department of Anaesthesiology and Critical Care Medicine, Faculty of Medicine and Biomedical Sciences, University of Yaoundé 1, Yaounde, Cameroon

${ }^{2}$ Department of General Medicine, Ibal Sub-Divsional Hospital, North West Region, Oku, Cameroon

${ }^{3}$ Department of General Medicine, Mayo Darle Sub-Divisional Hospital, Adamara Region, Banyo, Cameroon 
${ }^{4}$ Department of Surgery, National Social Insurance Fond Hospital, Yaounde, Cameroon

${ }^{5}$ Global Health System Solutions (GHSS) and Faculty of Sciences, University of Buea, Buea, Cameroon

${ }^{6}$ Department of Surgery and Sub-Specialties, Faculty of Medicine and Biomedical Sciences, University of Yaoundé 1, Yaounde, Cameroon

${ }^{7}$ Epidemiology and Biostatistics Unit, UCL Institute of Experimental and Clinical Research, Bruxelles, Belgium

Twitter Tianyi Tianyi Frank Leonel @tianyifrank1

Acknowledgements The authors thank the Liberian who designed the search strategy and identified the total number articles at the preliminary search.

Collaborators None.

Contributors JNT and CD conceived and designed the protocol. JNT drafted the manuscript. JNT, NVA, TTFL, AM, DAA and CD revised successive drafts of the manuscript. JNT is the guarantor of the review. All authors approved the final version of the manuscript.

Funding The authors have not declared a specific grant for this research from any funding agency in the public, commercial or not-for-profit sectors.

Competing interests None declared.

Patient consent for publication Not required.

Provenance and peer review Not commissioned; externally peer reviewed.

Open access This is an open access article distributed in accordance with the Creative Commons Attribution Non Commercial (CC BY-NC 4.0) license, which permits others to distribute, remix, adapt, build upon this work non-commercially, and license their derivative works on different terms, provided the original work is properly cited, appropriate credit is given, any changes made indicated, and the use is non-commercial. See: http://creativecommons.org/licenses/by-nc/4.0/.

\section{ORCID iDs}

Joel Noutakdie Tochie http://orcid.org/0000-0002-8338-2467

Ndip Valirie Agbor http://orcid.org/0000-0002-6708-6852

Celestin Danwang http://orcid.org/0000-0002-7976-4331

\section{REFERENCES}

1 Adesunkanmi ARK, Oseni SA, Adejuyigbe O, et al. Acute generalized peritonitis in African children: assessment of severity of illness using modified APACHE II score. ANZ J Surg 2003;73:275-9.

2 Drexel S, Tseng D. Primary Peritonitis: An Index Case of Mycoplasma hominis Infection in a Healthy Female. Case Rep Surg 2018;2018:1-4.

3 Skipworth RJE, Fearon $\mathrm{KCH}$. Acute abdomen: peritonitis. Surgery 2007;26:3.

4 Ghosh PS, Mukherjee R, Sarkar S, et al. Epidemiology of secondary peritonitis: analysis of 545 cases. Int J Sci Stud 2016;3:83-8.

5 Ross JT, Matthay MA, Harris HW. Secondary peritonitis: principles of diagnosis and intervention. BMJ 2018;361:k1407.

6 Strobel O, Werner J, Büchler MW. [Surgical therapy of peritonitis]. Chirurg 2011;82:242-8.

7 Nohr CW, Marshall DG. Primary peritonitis in children. Can J Surg 1984;27:179-81.

8 Sartelli M, Catena F, Ansaloni L, et al. Complicated intra-abdominal infections worldwide: the definitive data of the CIAOW study. World $J$ Emerg Surg 2014;9:37.
9 Chichom-Mefire A, Fon TA, Ngowe-Ngowe M. Which cause of diffuse peritonitis is the deadliest in the tropics? A retrospective analysis of 305 cases from the south-west region of Cameroon. World J Emerg Surg 2016;11:14.

10 Mishra SP, Tiwary SK, Mishra M, et al. An introduction of tertiary peritonitis. J Emerg Trauma Shock 2014;7:121-3.

11 Anaya DA, Nathens AB. Risk factors for severe sepsis in secondary peritonitis. Surg Infect 2003;4:355-62.

12 Gupta H, Dupuy DE. Advances in imaging of the acute abdomen. Surg Clin North Am 1997;77:1245-63.

13 Martin RF, Rossi RL. The acute abdomen. An overview and algorithms. Surg Clin North Am 1997;77:1227-43.

14 Gauzit R, Péan Y, Barth X, et al. Epidemiology, management, and prognosis of secondary non-postoperative peritonitis: a French prospective observational multicenter study. Surg Infect 2009;10:119-27.

15 Bali RS, Verma S, Agarwal PN, et al. Perforation peritonitis and the developing world. ISRN Surg 2014;2014:1-4.

16 Sartelli M, Abu-Zidan FM, Labricciosa FM, et al. Physiological parameters for prognosis in abdominal sepsis (PIPAS) study: a WSES observational study. World J Emerg Surg 2019;14:34.

17 Ugochukwu Al, Amu OC, Nzegwu MA. Ileal perforation due to typhoid fever - review of operative management and outcome in an urban centre in Nigeria. Int J Surg 2013;11:218-22.

18 Sanogo ZZ, Camara M, Doumbia MM, et al. [Digestive tract perforations at Point G Teaching Hospital in Bamako, Mali]. Mali Med 2012;27:19-22.

19 Adesunkanmi ARK, Badmus TA. Pattern of antibiotic therapy and clinical outcome in acute generalized peritonitis in semi-urban and rural Nigerians. Chemotherapy 2006;52:69-72.

20 Johnson CC, Baldessarre J, Levison ME. Peritonitis: update on pathophysiology, clinical manifestations, and management. Clinical Infectious Diseases 1997;24:1035-45.

21 Akoh JA. Peritoneal dialysis associated infections: an update on diagnosis and management. World J Nephrol 2012;1:106.

22 Søreide K, Thorsen K, Søreide JA. Strategies to improve the outcome of emergency surgery for perforated peptic ulcer. Br J Surg 2014;101:e51-64.

23 Moher D, Liberati A, Tetzlaff J, et al. Preferred reporting items for systematic reviews and meta-analyses: the PRISMA statement. PLoS Med 2009;6:e1000097.

24 Moher D, Shamseer L, Clarke M, et al. Preferred reporting items for systematic review and meta-analysis protocols (PRISMA-P) 2015 statement. Syst Rev 2015;4:1.

25 Tochie J, Agbor VN, Tianyi F-L, et al. Global epidemiology of acute generalized peritonitis: a protocol for a systematic review and metaanalysis. Available: https://www.crd.york.ac.uk/prospero/display_ record.php?ID=CRD42019143331 [Accessed 15 Nov 2019].

26 Viera AJ, Garrett JM. Understanding interobserver agreement: the kappa statistic. Fam Med 2005;37:360-3.

27 The Joanna Briggs Institute. The Joanna Briggs Institute critical appraisal tools for use in JBI systematic reviews: checklist for prevalence studies, 2017. Available: http://joannabriggs.org/ research/critical-appraisal-tools.html [Accessed 15 Feb 2019].

28 DerSimonian R, Laird N. Meta-Analysis in clinical trials. Control Clin Trials 1986;7:177-88.

29 Barendregt JJ, Doi SA, Lee YY, et al. Meta-Analysis of prevalence. $J$ Epidemiol Community Health 2013;67:974-8.

30 Cochran WG. The combination of estimates from different experiments. Biometrics 1954;10:101-29.

31 Higgins JPT, Thompson SG. Quantifying heterogeneity in a metaanalysis. Stat Med 2002;21:1539-58.

32 Egger M, Davey Smith G, Schneider M, et al. Bias in meta-analysis detected by a simple, graphical test. BMJ 1997;315:629-34. 